Revista Brasileira de Engenharia Biomédica,

v. 26, n. 1, p. 49-54, abril 2010

(C) SBEB - Sociedade Brasileira de Engenharia Biomédica

ISSN 1517-3151

http://dx.doi.org/10.4322/rbeb.2012.080

Comunicação Técnica

Recebido em 20/07/2009, aceito em 22/03/2010

\section{Prótese para controle de fluxo esofagiano como nova técnica para o tratamento da obesidade}

\section{Prosthesis for flow control in the esophagus as a new technique for the treatment of obesity}

\author{
Suélia de Siqueira Rodrigues Fleury Rosa* \\ Adson Ferreira da Rocha \\ Lourdes Mattos Brasil \\ Edgard Costa Oliveira \\ Patrícia Mendonça Carlos de Paula \\ Faculdade UnB Gama, FGA / UnB \\ Área Especial 2, Lote 14, Setor Central, CP 8114 \\ 72405-610 Gama, DF - Brasil \\ E-mail: rodrigues.suelia@gmail.com
}

\section{José da Conceição Carvalho}

Departamento Ortopedia e Cirurgia Plástica - FM Hospital de Clínicas, UFG

$1^{a}$ Avenida, $\mathrm{s} / \mathrm{n}$ - Setor Leste Universitário

74605-020 Goiânia, GO - Brasil

*Autor para correspondência
Resumo

O presente artigo apresenta uma invenção desenvolvida para auxílio à redução e ao controle da obesidade, embasado no controle do fluxo de alimentos no esôfago. Neste novo método proposto, um dispositivo colocado no esôfago diminui seu raio efetivo, reduzindo, por consequência, a velocidade e o fluxo com que o alimento pode ser consumido. A base do método é um dispositivo denominado módulo de látex, com formato aproximadamente cilíndrico, que é aplicado no esôfago. Este módulo contém um lúmen central, com diâmetro consideravelmente menor que o diâmetro do esôfago, que limita a passagem de alimento pelo órgão. Na concepção do método e do módulo, buscouse realizar o controle de fluxo alimentar através do esôfago, sem causar agressões ao tecido, e sem comprometer a digestão e absorção dos alimentos. Desenvolvido a partir do látex natural, extraído da seringueira Hevea brasiliensis, tal módulo foi avaliado quanto a características construtivas e mecânicas, aplicabilidade, facilidade de remoção, eficácia na redução de fluxo, peso e danos ao esôfago. O módulo foi posicionado no esôfago por meio de vídeo-endoscopia em um cão piloto e manteve-se no local por sete dias, nos quais foi acompanhado por meio de radiografias sucessivas até a sua retirada, momento em que foi avaliada a integridade da mucosa esofágica. Os resultados apresentados demonstram que o método proposto é uma nova possibilidade de controle e redução da obesidade em pessoas e animais.

Palavras-chave: Látex, Obesidade, Controle de fluxo.

\section{Abstract}

This work introduces a device to aid in the treatment of obesity by controlling the flow of substances in the esophagus. In the method proposed, a device placed in the esophagus reduces its effective radius, thus reducing the flow and speed in which the food can be consumed. The basis of the method is a nearly cylindrical latex module, which is applied in the esophagus. The module's central lumen diameter is considerably smaller than the diameter of the esophagus. This limits the rate of food passage through the esophagus. The device was designed in such a way that it could control the food flow through the esophagus without causing aggression to its internal tissue and without compromising the digestion and absorption of food. The device was built using natural latex from the rubber tree Hevea brasiliensis, and was evaluated according to its construction, mechanical properties, applicability, effectiveness in reducing food flow, weight, and damage to the esophagus. The module was placed in the esophagus by means of video-endoscopy and remained in place for seven days, wherein it was accompanied by successive radiographies until its withdrawal by video-endoscopy, when we were able to examine the integrity of the esophagus wall. The results presented in this paper show that the proposed method is a new possibility for the treatment of obesity in humans and animals.

Keywords: Latex, Obesity, Flow control. 


\section{Extended Abstract}

\section{Introduction}

In humans, food ingestion is influenced by non-biological variables that include social, economic, and cultural factors, as well as convenience and meal day schedule. However, physical and biological factors such as sight, smell, taste and the metabolic state of the organism regulate appetite. In this article we propose a module for Esophageal Flow Control (EFC), which is a new device for controlling weight that is applied to the esophagus without altering the digestive tract. The EFC promotes the reduction of food flow, while preserving all the physiological processes of digestion. The module was constructed naturally using rubber latex (Hevea brasiliensis) and, thus, it is waterproof, flexible and easy to apply and remove. The goal of this research was to develop a prototype of the module and to test it in a medium-sized canine, in a 7-day period. The experiment allowed for preliminary testing of the technique as well as identifying the module's shortcomings. These results should be useful for further development of the module.

\section{Materials and Methods}

The raw material chosen for the experiment was extracted from natural rubber latex. From this natural latex, a final compound was prepared to give the EFC module its essential characteristics of elasticity, smoothness, as well as waterproof and hypoallergenic qualities. This compound was reached by adding chemicals, in accordance to the recommendations of Mrue (1996). It was designed to fit the anatomy and characteristics of a dog's esophagus wall tissue, especially the longitudinal grooves of the esophageal mucous membrane (Mrué, 1996). It has three distinct elements: the external and internal tubes and the valve. The testing procedure was approved by the committee of ethics in research at the Federal University of Goiás, Brazil. The animal was previously subjected to the procedures for vaccination, control of ecto and endoparasites, in addition to laboratory tests, hematology and clinical chemistry, for the verification of its health conditions. After loss of reflexes, the animal was subjected to endoscopic examination, initially to assess the physiological macroscopic conditions and anatomy of the esophagus. Then, the deflated EFC module was placed. The module was introduced slowly and positioned $3 \mathrm{~cm}$ below the upper following the placement of the module, the behavior of the animal was evaluated by a veterinarian in terms of food ingestion, vomiting, coughing and loss of body weight with observations recorded daily. In order to confirm the continuation of the module's positioning, radiological exams were performed on the latero-side incidence of the right cervical region immediately after the placement of the module and then again two days before the removal of the module. The removal of the module followed a procedure similar to the one used for its introduction inside the esophagus.

\section{Results and Discussion}

The dog was submitted to a food restriction of 12 hours as a prophylaxis for vomiting in the trans-anesthetic stage, because could interrupt the endoscopy procedure and cause discomfort to the animal. Moreover, this procedure prevents from a potential cause of development of pneumonia caused by aspiration. The result of the application methodology through endoscopy was positive because it allowed the evaluation of the integrity of the esophagus prior to the introduction of the module. Also, the use of this technique allowed the installation of the module by slow and monitoring for accurate positioning without damaging the mucous membrane. Through video-endoscopy, the module in the esophagus was shown on the computer screen, reducing its diameter from $3 \mathrm{~cm}$ to $1 \mathrm{~cm}$. Along the seven days following the placement of the module, its retention within the esophagus was accompanied by $x$-ray examinations. After seven days, the animal was again subjected to a video-endoscopy exam for removing the module. The dog had a weight loss of about $4 \%$ during one week of observation. However, this result should be carefully checked and revalidated in further studies by using the same approach. Nonetheless, these preliminary results are encouraging and are a good justification for testing this new method in a larger group of dogs in order to track other parameters related to the module and the physiology of the animal.

\section{Conclusions}

The use of the esophageal flow control module caused no apparent damage to or changes in the dog's esophagus wall. Moreover, it promoted weight loss in the animal without impairing the physiological parameters or causing apparent behavioral changes. 


\section{Introdução}

Nos dias atuais, a obesidade tem sido cada vez mais reconhecida como uma doença séria, responsável pela morbidade de milhões de pessoas, e que desencadeia problemas individuais relacionados ao status psicossocial e à qualidade de vida (Fontaine et al., 1997). A Organização Mundial de Saúde (OMS), por sua vez, a classifica como um problema de saúde pública mundial e como uma enfermidade crônica, já catalogada no Código Internacional de Doenças (CID-10) como E-66. Embora a ciência tenha descoberto e esclarecido algumas teorias conflitantes e técnicas de tratamento de tal patologia, este é um assunto com muitas pesquisas a serem realizadas, pois, pelo que se observa na literatura, existem informações inexploradas e contraditórias. Atualmente, existem diversas formas para tratamento encontradas na literatura, incluindo dietas, tratamentos farmacológicos e não-farmacológicos e cirurgias. Entretanto, em todos os métodos há um ponto comum referente à diminuição da ingestão alimentar, pois para que haja perda de peso é necessário que ocorra um déficit energético. Além disso, todos os métodos apresentam vantagens e desvantagens associadas à forma de intervenção para a perda de peso. Entre as técnicas disponíveis para o tratamento da obesidade, as mais eficazes na redução de ingestão alimentar são as técnicas cirúrgicas. Isso ocorre devido à restrição mecânica e física que elas impõem ao paciente. Entre as três formas básicas de tratamento cirúrgico destacam-se as seguintes: 1) técnicas disabsortivas: cirurgia de Scopinaro e duodenal switch; 2) técnicas restritivas - balão intragástrico, gastroplastia vertical com bandagem (cirurgia de Mason) e banda gástrica ajustável; 3) técnicas mistas - cirurgia de Fobi-Capella. Dentre as técnicas citadas, a denominada balão intragástrico (Bioenterics Intragastric Balloon-BIB ${ }^{\circledR}$ ), que possui o mesmo princípio da que estamos propondo, tem como elemento principal uma prótese de silicone, de formato esférico e de superfície lisa, que apresenta uma válvula por onde o balão é insuflado, dentro do estômago do paciente. A presença do balão dentro do estômago causa uma sensação de plenitude (estômago cheio), ou seja, a chamada saciedade precoce. Uma desvantagem do $\mathrm{BIB}^{\circledR}$, com gás ou soro, reside no fato de que este constitui-se em um dispositivo de utilização temporária (no máximo seis meses), fazendo-se necessária a retirada do balão, pois o mesmo é lentamente corroído pelos ácidos estomacais. A pesquisa bibliográfica revelou que não foram encontradas, ainda, técnicas ideais para o tratamento e controle do peso que demonstrassem um índice completamente satisfatório (em relação às variáveis de perda, manutenção e velocidade) de sucesso.

$\mathrm{O}$ objetivo da presente pesquisa foi $\mathrm{o}$ desenvolvimento de uma nova técnica de tratamento e de um dispositivo inédito, denominado módulo para Controle de Fluxo Esofagiano $\left(\mathrm{CFE}^{\circledR}\right)$, com registro de patente PI0803231-9, feito de material biossintético à base de látex natural (extraído da seringueira Hevea brasiliensis), para ser aplicado por meio da técnica endoscópica no esôfago, para controlar e limitar a velocidade e o volume da ingestão alimentar e, consequentemente, proporcionar a redução de peso (Rodrigues, 2008). Para validar a aplicabilidade e o modelo proposto, foram realizadas análises e testes de aplicação e adaptabilidade em um cão.

\section{Materiais e Métodos}

$\mathrm{O}$ módulo $\mathrm{CFE}^{\circledR}$ foi desenvolvido em quatro etapas principais: confecção, caracterização, aplicação e validação do módulo. No desenvolvimento do módulo, foram levados em conta requisitos indispensáveis a um produto aplicado no interior do esôfago, tais como elasticidade, suavidade, resistência, impermeabilidade ehipoalergenicidade. Por este motivo, escolheu-se como matéria prima para o desenvolvimento do módulo o "látex natural", extraído da Hevea brasiliensis.

No processo de confecção do módulo, utilizouse a técnica de banhos sucessivos de imersão (Mrué, 1996), que consiste na introdução dos moldes no interior do composto final de látex, em posição perpendicular ao plano, de forma gradual e uniforme, seguido de aquecimento em estufa termostatizada. A caracterização do módulo foi feita por meio de análises sistêmicas de algumas de suas propriedades construtivas, estruturais, térmicas e de teste experimental em cão piloto. $\mathrm{Na}$ análise das propriedades construtivas, foi realizada inspeção visual para detecção de eventuais defeitos de modelagem e montagem, associados, por exemplo, ao formato da superfície, das ranhuras, da conexão da válvula e do fio, por meio da utilização de paquímetro e régua.

Para se avaliar as propriedades estruturais, foram realizados testes para verificação de vazamentos, resistência da parede externa, capacidade da válvula e uniformidade da distribuição do gás. Para isso, foi utilizado um equipamento microcontrolado, especialmente projetado para inflar o módulo com pressão exata. As pressões usadas foram definidas com base na pressão de aplicação que será utilizada 
no módulo e fundamentadas na revisão bibliográfica realizada neste estudo. Os testes foram feitos a partir dos procedimentos listados a seguir: 1) vazamento; 2) ruptura; 3) uniformidade. Na análise de resistência térmica foi utilizada uma estufa termostatizada. Os corpos de provas foram confeccionados seguindo o fluxograma de preparo (mesmo número de banhos e tempo de vulcanização) e possuíam as dimensões do produto, com tolerância de $0,2 \mathrm{~mm}$ para diâmetro e $0,5 \mathrm{~mm}$ para comprimento. As amostras foram acondicionadas em uma superfície lisa e plana de vidro, à temperatura de $23^{\circ} \mathrm{C}$, com massa de amostra igual a $28 \pm 0,2 \mathrm{~g}$. As corridas foram realizadas no intervalo da temperatura de $20^{\circ} \mathrm{C}$ a $300{ }^{\circ} \mathrm{C}$, com razão de aquecimento de $5{ }^{\circ} \mathrm{C} / \mathrm{min}$ (ASTM, 1998). Para verificação da massa, utilizou-se a balança analítica eletrônica, com capacidade máxima de 210 g, e precisão de 0,1 mg. Para avaliação das propriedades mecânicas, foi usada a técnica dinâmico-mecânica no modo de elasticidade e resistência: estes foram definidos por serem preferenciais para medidas de borracha por meio de ensaios normalizados pela ASTM (1998). Por isso, na análise das propriedades físicas buscou-se avaliar o módulo de Young, aplicando-se tensões em sentido longitudinal e transversal para se obter o índice. A técnica utilizada foi a análise dinâmico-mecânica (DMA), que é uma técnica na qual as respostas mecânicas de uma amostra, sob carga, são monitoradas em relação ao tempo. As forças de tensão foram aplicadas na parte externa do módulo, que era a região de interesse - é a que se expande. A força de tensão, em vista disso, foi aplicada em ordem crescente, até que ocorresse a ruptura da amostra. Então, amostras com aproximadamente $10 \mathrm{~mm}$ de espessura e $1 \mathrm{~cm}^{2}$ de seção transversal foram retiradas do módulo $\mathrm{CFE}^{\circledR}$.

O teste piloto como o módulo $\mathrm{CFE}^{\circledR}$ foi realizado com um cão, obedecendo-se os princípios e as normas éticas de protocolo de pesquisa em animais. $\mathrm{O}$ protocolo experimental foi previamente submetido e aprovado pelo Comitê de Ética em Pesquisa Médica Humana e Animal da Universidade Federal de Goiás (processo de número 060/2008, com cadastro no SAPP-WEB sob número 33256). O uso experimental em um animal foi proposto para ser realizado por um período de sete dias, visando à obtenção de dados importantes, tais como dimensão mais adequada, comprimento, tipo de ranhuras, capacidade de fixação sem intervenção para serem aplicados na confecção do módulo. Além de se estudar o procedimento de aplicação e remoção do módulo controlador de flu- xo, foram avaliados diversos aspectos: peso corporal; comportamento do animal; alimentação; tempo de permanência do módulo no esôfago; danos à parede do esôfago e complicações imprevistas. O módulo foi inserido por endoscopia em um cão macho, adulto, de raça mista, com peso corporal de $12,7 \mathrm{~kg}$. A aplicação do módulo foi precedida por jejum alimentar de 12 horas. Na pré-anestesia, utilizou-se diazepam na dose de $0,5 \mathrm{mg} \cdot \mathrm{kg}^{-1}$, e na anestesia foi utilizado propofol, com 6,0 mg. $\mathrm{kg}^{-1}$, para indução, e 0,5 mg. $\mathrm{kg}^{-1}$. min, para manutenção, todos administrados por via intravenosa. $\mathrm{O}$ módulo foi introduzido lentamente e posicionado entre a segunda e a quarta vértebras cervicais (3 $\mathrm{cm}$ abaixo do esfíncter superior esofágico). Atingida a posição desejada, o módulo foi inflado lentamente até que atingisse a medida de pressão especificada em $60 \mathrm{mmHg}$. Posteriormente, o animal recebeu dieta na forma líquido-pastosa. O comportamento do animal foi avaliado nos sete dias subsequentes à colocação do módulo em relação aos seguintes parâmetros: ingestão de alimento, mímica de vômito, tosse e perda de peso corporal. Para confirmar a permanência de posicionamento do módulo, foram realizados exames radiográficos da região cervical em incidência látero-lateral direita, imediatamente após a colocação do módulo e a cada dois dias, até a retirada do módulo. A remoção do módulo seguiu o mesmo protocolo citado para a colocação.

\section{Resultados}

Na Figura 1 pode-se observar o aspecto morfológico macroscópico do módulo CFE ${ }^{\circledR}$ desenvolvido.

As características macroscópicas do módulo são apresentadas na Tabela 1.

Os testes de vazamento foram satisfatórios para as pressões de aplicação utilizada. Com relação aos testes de resistência da parede externa quando o módulo foi submetido à pressão-teste de $240 \mathrm{mmHg}$, não se verificou a ocorrência de nenhuma ruptura das
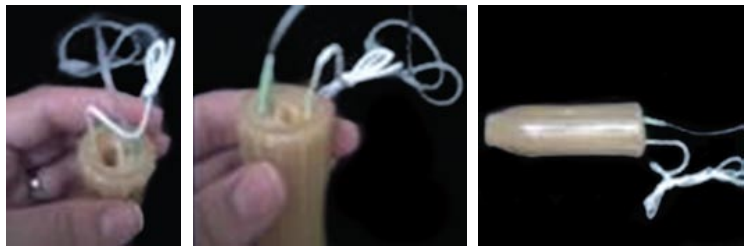

Figura 1. Fotografia do módulo controlador de fluxo esofagiano - $\mathrm{CFE}^{\oplus}$; da esquerda para direita: vistas superior e laterais. Figure 1. Photo of the module of esophagus flow control - CFE ${ }^{\oplus}$; right to the left: side and upper view. 
Tabela 1. Características e dimensões do módulo CFE ${ }^{\oplus}$. Table 1. Characteristics and dimensions of the CFE ${ }^{\circledR}$ module.

\begin{tabular}{ccc}
\hline Classificação & Características & Dimensão \\
\hline Formato & Cilíndrico & $2,5 \mathrm{~cm}$ de diâmetro externo \\
Superfície Externa & Ondulada com ranhuras longitudinais & $1,5 \mathrm{~mm}$ de espessura \\
Superfície Interna & Plana & 12 ranhuras de 7,0 mm de saliência e \\
Cor & & $5,0 \mathrm{~mm}$ de espaço entre elas. \\
Comprimento & Amarela & $1,0 \mathrm{~cm}$ diâmetro do furo \\
Fio fixação & - & $0,2 \mathrm{~cm}$ de espessura \\
& Fio cirúrgico & - \\
\hline
\end{tabular}

paredes externas e nem das estruturas que compõem o módulo. A uniformidade da distribuição do gás para o intervalo de pressão definido de aplicação do módulo (60 $\mathrm{mmHg}$ até $120 \mathrm{mmHg}$ ) não acarretou alteração aparente no módulo no que se refere a sua uniformidade. Entretanto, a medida construtiva de diâmetro externo apresentou alguma variação, sendo que esta permaneceu dentro do intervalo aceitável (0,15 mm). A análise térmica da vulcanização do látex evidenciou que o látex possui duas etapas de perda de massa, sendo que a primeira, que se estende até aproximadamente $160^{\circ} \mathrm{C}$ é mais gradual e constante. De acordo com a literatura estudada (D'Auzac e Jacob, 1989), essa etapa se caracteriza pela perda de água, amônia e subprodutos dos componentes não-borrachosos presentes (proteínas, aminoácidos, carboidratos, lipídios, ácidos nucléicos). A segunda etapa, que ocorre entre $240^{\circ} \mathrm{Ce} 300^{\circ} \mathrm{C}$, foi caracterizada como a principal fase de degradação do dispositivo. Com esta análise, pode-se sugerir, como margem de trabalho para confecção do módulo $\mathrm{CFE}^{\circledR}$, uma faixa de temperatura de $70{ }^{\circ} \mathrm{C}$ a $110^{\circ} \mathrm{C}$, sem que se percam as características básicas do material aqui proposto. Os resultados obtidos nos ensaios mecânicos foram: tensão de rotura de 2,28 N/m² e 15,63 N/m², e módulo de Young $0,09 \cdot 10^{5} \mathrm{~N} / \mathrm{m}^{2}$ e $0,33 \cdot 10^{5} \mathrm{~N} / \mathrm{m}^{2}$, longitudinal e transversal, respectivamente. $\mathrm{O}$ resultado da metodologia de aplicação via endoscopia foi positivo, pois permitiu a avaliação da integridade da mucosa previamente à colocação do módulo. Além disso, o uso dessa técnica facilitou a instalação do módulo de forma lenta e monitorada permitindo o posicionamento preciso e sem danos à mucosa. Nos sete dias subsequentes à colocação do módulo, sua retenção dentro do esôfago foi acompanhada por meio de exames radiográficos e verificou-se sua perfeita acomodação entre a segunda e quarta vértebras cervicais. Nas primeiras 15 horas constatouse salivação, tosse espaçada e episódio de engasgo moderado. Tais ocorrências possivelmente estiveram relacionadas ao período de acomodação do esôfago ao objeto inserido em seu lúmen. Após a primeira refeição, foi realizado exame radiográfico para verificação da permanência de posicionamento do módulo $\mathrm{CFE}^{\circledR}$. $\mathrm{O}$ resultado do exame confirmou favoravelmente as expectativas, indicando, entre outros aspectos, que a consistência do alimento foi adequada para passar através da porção interna do módulo sem empurrálo caudalmente. $\mathrm{O}$ animal foi avaliado a cada duas horas quando foram então analisados sinais vitais, tais como: respiração e frequência cardíaca; análise da excreção - urina e fezes; e análise comportamental. Os dados serviram de comparação com os resultados obtidos em 10 dias de tratamento preparatório antes da colocação do módulo. Nas análises não houve alterações significativas após o tempo de acomodação do módulo. As frequências cardíacas mantiveramse na média em comparação com o período anterior e houve uma alteração nos primeiros dois dias de comportamento do animal, o qual estava menos ativo nesta ocasião. Esses resultados permitem inferir que as dimensões do módulo, a pressão exercida sobre as paredes do esôfago e o local de instalação do mesmo foram adequadas. No transcorrer do exame, não foi observada presença de restos alimentares na porção cranial do esôfago ou no entorno do módulo. Após a remoção do módulo, não se constatou, no exame com vídeo-endoscopia, danos à parede do esôfago em toda sua extensão.

\section{Discussão}

Neste ponto, é necessário reafirmar que a obesidade é uma doença que afeta um indivíduo como um todo e os fatores que causam a obesidade são muitos, mas em geral a maior causa da obesidade ainda é o comportamento alimentar alterado e/ou inadequado. Em vista de tal realidade, foi apresentado, no presente artigo, um novo dispositivo para o tratamento de 
tais casos, denominado "módulo controlador de fluxo esofagiano" $\left(\mathrm{CFE}^{\circledR}\right)$. Destaca-se que o método é totalmente original e inédito, desenvolvido com um componente natural látex, extraído da Hevea brasiliensis. A base desta proposta foi a redução da ingestão alimentar. Embora o estudo da variável perda de peso não tenha sido, nesse momento da pesquisa, o alvo principal desta etapa de teste do módulo, constatou-se que ocorreu diminuição da ingestão alimentar do cão. Vale ressaltar que o cão foi tratado nos dez dias que antecederam o experimento com a mesma alimentação e cuidados, e que a perda de peso só foi observada durante o experimento. Isso é um indício de que a perda de peso está associada à presença do módulo e não ao tipo de alimentação administrada. Os resultados preliminares são encorajadores e incentivam a verificação desse novo método em um grupo maior de cães para que se faça o monitoramento de diversos outros parâmetros relacionados ao módulo e à fisiologia do animal.

\section{Conclusão}

Com base nos resultados obtidos, conclui-se que o módulo $\mathrm{CFE}^{\circledR}$ tem aplicabilidade admissível, ficando demonstrado, assim, que o material utilizado e o modelo proposto apontam para uma perspectiva de se obter uma metodologia com potencial multidisciplinar para o tratamento da obesidade em humanos.

\section{Agradecimentos}

Ao Universia e ao Banco Santander-que patrocinaram parcialmente o projeto através do Prêmio Santander de Biotecnologia do ano de 2008. À CAPES, pelo financiamento por meio de bolsa de doutorado, e ao $\mathrm{CNPq}$, pelo apoio por meio de bolsa de produtividade em pesquisa.

\section{Referências}

AMERICAN SOCIETY FOR TESTING AND MATERIALS - ASTM. Livro de Normas ASMT de 1997, Parte 9.1: Rubber, Carbon Black, Gaskets. 1998.

D'AUZAC, J.; JACOB, J. L. Composition of Latex from Hevea brasiliensis as a lacticiferous cytoplam. In: D'AUZAC, M.; JACOB, J. L.; CHRESTIN, H. (Eds.). Physiology of Rubber Tree Latex, Florida: CRC Press, 1989. p. 59-96.

FONTAINE, K. R.; BAROFSKY, I.; CHESKIN, L. J. Predictors of quality of life for obese persons. Journal of Nervous and Mental Disease, v. 185, n. 2, p. 120-122, 1997.

MRUÉ, F. Substituição do Esôfago Cervical por Prótese Biossintética de Látex: estudo experimental em cães. 1996. 114 p. Dissertação (Mestrado) - Faculdade de Medicina - FMRP, Universidade de São Paulo - USP, Ribeirão Preto, 1996.

RODRIGUES, S. S. Desenvolvimento de um sistema de controle de fluxo esofagiano para tratamento da obesidade. 2008. 106 p. Tese (Doutorado) - Universidade de Brasília - UnB, Brasília, DF, 2008. 